\title{
PONDERACIÓN DE DERECHOS FUNDAMENTALES EN ÉPOCA DE PANDEMIA
}

\section{WEIGHTING OF FUNDAMENTAL RIGHTSIN TIME OF PANDEMIC}

AROLDO RAMIRO AGUIRRE NÚÑEZ*

* Juez del $6^{\circ}$ Juzgado Penal Unipersonal de Lima Norte, Maestro en Derecho Penal, por la UNFV, Docente Principal de la Academia de la Magistratura

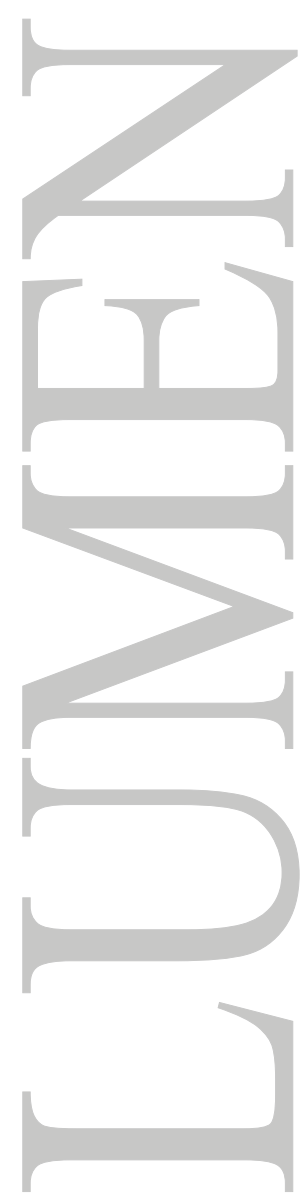




\title{
PONDERACIÓN DE DERECHOS FUNDAMENTALES EN ÉPOCA DE PANDEMIA
}

\author{
WEIGHTING OF FUNDAMENTAL RIGHTS IN TIME OF PANDEMIC
}

Aroldo Ramiro Aguirre Núñez

\begin{abstract}
RESUMEN:
La aparición e instalación en nuestro país de la pandemia provocada por el llamado covid-19, ha provocado la emisión de distintos decretos supremos, que en nombre del resguardo de la salud, el medio de la familia y la comunidad, han recortado de un modo intenso determinados derechos fundamentales de primer grado, como lo son la libertad de tránsito y la libertad del trabajo. El argumento normativo empleado por el Poder Ejecutivo es débil, pues la preeminencia de ciertos derechos fundamentales de segundo grado por encima de los otros llamados derechos fundamentales de primer grado supone un escenario hipotético de inminente controversia constitucional en la que habrá de emplearse la llamada ponderación de derechos fundamentales, en los que se debatirán los principios de idoneidad, necesidad y ponderación o proporcionalidad propiamente dicha, cuyos escenarios devienen ser muy interesantes y sobretodo promisorios para el esclarecimiento del conflicto de derechos.
\end{abstract}

\section{ABSTRACT:}

The appearance and installation in our country of the pandemic caused by the so-called covid-19, has led to the issuance of various supreme decrees, which in the name of protecting the health, the environment of the family and the community, have cut in a way Intensive certain fundamental rights of the first degree, such as freedom of transit and freedom of work. The normative argument used by the Executive Power is weak, since the preeminence of certain fundamental rights of the second degree over the other so-called fundamental rights of the first degree supposes a hypothetical scenario of imminent constitutional controversy in which the so-called weighting of fundamental rights, in which the principles of suitability, necessity and weighting or proportionality itself will be debated, whose scenarios become very interesting and above all promising for the clarification of the conflict of rights.

\section{PALABRAS CLAVE:}

Pandemia, decreto supremo, afectación, derechos fundamentales, idoneidad, necesidad, ponderación, proporcionalidad, argumentación, derecho.

\section{KEY WORDS}

Pandemic, supreme decree, affectation, fundamental rights, suitability, necessity, weighting, proportionality, argumentation, law.

\section{INTRODUCCIÓN}

La súbita y reciente aparición en nuestro país del llamado covid-19, producto de la gran pandemia mundial, con mortíferos efectos en los distintos países del mundo, ha obligado al poder ejecutivo de nuestro país al uso de la facultad constitucional para la emisión de sucesivos Decretos Supremos, decretando el estado de emergencia y extremas medidas de cuarentena obligatoria sin precedentes en nuestra historia reciente. Estas medidas, motivadas seguramente por el propósito de tutelar la vida de los pobladores del país, y el propósito de evitar la expansión del mal a los confines de nuestra geografía, han originado de un modo casi inmediato un verdadero desastre económico en el país, que se ha manifestado por la pérdida de innumerables puestos de trabajo, sobretodo de la 
población vulnerable o informal de nuestra realidad, habiéndose producido lo que los economistas denominan un primer ciclo de recesión económica, luego de largos años de continuo crecimiento económico de nuestro país. Es decir, la pandemia del covid-19, apenas instalada en nuestro país, ha ocasionado desde los primeros días de su ominosa confirmación, un brusco frenazo de la locomotora de la economía, amenazando incluso con descarrillar todo el tren de nuestra vida cotidiana, la de una gran masa mayoritaria de pobladores del país, llevando al traste los años de sacrificio que ha costado edificar esta realidad sólo en apariencia sólida, si es que no se actúa con responsabilidad y desprendimiento a la hora de tomar las decisiones económicas más importantes como necesaria respuesta a esta súbita e inesperada realidad.

Ya se había producido en nuestra realidad la llamada recesión económica, caracterizada, por dos trimestres consecutivos de crecimiento negativo de la economía, el primero de ellos -enero, febrero y marzo- ya transcurrido y verificado además por cifras que se aprestan a ser oficiales, y el sub siguiente ciclo, -abril, mayo y junio- en seguro camino de suceder lo mismo, con peores cifras todavía, agregando a este comentario que precisamente el último decreto supremo del ejecutivo que ha prolongado el estado de emergencia y la cuarentena obligatoria hasta el último día de junio de este año 2020, no hace sino confirmar esta sombría proyección de un modo objetivo. (Gonzales J. 2020).

Es decir que, el fenómeno externo, inusual y súbito de la pandemia del covid-19, que llegó a instalarse sin ninguna invitación a nuestro medio, ha provocado la repentina detención y luego el retroceso de la actividad económica del país, sin que sea todavía posible por ahora, cuantificar los efectos de su trascendencia negativa a futuro.

La pandemia, además, viene exponiendo, esta vez a plena luz del día y de un modo descarnado, la consabida insuficiencia de la infraestructura de ciertos sectores críticos del país, comenzando por mencionar el de salud, que a estas horas luce no solamente desfasada, sino incluso dramáticamente insuficiente. Sólo para recordar por un momento, no están muy distantes aún los sucesivos reclamos del gremio médico del país, reflejadas en no pocas huelgas de años recientes de las que nos enteramos ya sea por los medios de comunicación o por la imposibilidad de poder acceder a los servicios básicos de salud. Esta no es sino una pequeña muestra de la gran paradoja en la que habíamos incurrido al mostrar muy complacidos ante el mundo, nuestra apreciable cifra de las reservas internacionales, muy superior a las de algunos países vecinos, que si bien le ha otorgado entre otros solidez a nuestro signo monetario y un clima de estabilidad y predictibilidad para los negocios, pero que sin embargo, llegada la hora de la verdad y de mano de la pandemia, ahora nos acaba de extender la ominosa factura por los largos años de irresponsable postergación de esta sector, a pesar que si se pudo realizar con los recursos que siempre supimos que existen y que en buena cuenta implica en no cumplimiento por parte del Estado de su obligación por velar adecuadamente por la salud de los pobladores del país.

Este escenario surrealista, propio de una realidad que nadie hubiera podido imaginar o siquiera suponer para nuestra época, nos ha devuelto de un súbito empujón a escenarios de la edad media que hasta nos parecían tan distantes y tan lejanas que sólo las creíamos producto del relato en los libros de historia universal, sin olvidar en modo alguno la gran catástrofe poblacional que representó para nuestro país, el inesperado encuentro de dos mundos que sucedió hace ya casi 500 años atrás, tal como también lo registra nuestra propia historia. La gran diferencia actual, es que este dramático evento, ahora tiene implicancias y alcance global, mundial, como un fruto amargo del progresivo acortamiento de las distancias y el inusitado intercambio de productos y personas que se registra hasta fechas recientes, y a donde se agrega como un amargo añadido, la creciente actividad turística a nivel mundial. 
Esta súbita realidad, que de pronto vino acompañada por las cifras de los efectos perniciosos en otros países, ha provocado de un modo inmediato una agresiva intervención del Estado, que ha ordenado a limitar mediante decreto y en forma ostensible diversos derechos fundamentales, tales como la libertad para transitar o el derecho a trabajar precisamente. Pues bien, antes estos recortes explícitos de derechos fundamentales, curiosamente no se verifica todavía, por lo menos hasta la fecha, ningún reclamo por las vías constitucionales correspondientes (acción de amparo o hábeas corpus). ¿Se ha resignado la población a sufrir una cuarentena, que la obliga a estar confinada dentro de sus viviendas?. Esta es una respuesta que no cabe conjeturar todavía, no solamente porque si bien no apreciamos un pánico generalizado por el virus presente en nuestra realidad, sino más bien un natural temor de perder la vida, por lo que prefiere, por ahora, esperar todo el apoyo asistencial posible, por parte del Estado.

El derecho fundamental que determina la libertad de tránsito o el derecho fundamental a trabajar, se hallan recortados aparentemente por la preeminencia del otro derecho fundamental a preservar la vida de los ciudadanos, y que se pretende tutelar mediante la cuarentena decretada a nivel nacional. Sin embargo, este sencillo razonamiento, no hace sino reflejar la aplicación de la llamada ponderación de derechos fundamentales, que es donde finalmente habrá de residir, probablemente, la motivación que pretenda justificar o no las decisiones que han recortado otros derechos fundamentales, previstos y sancionados en la Constitución Política vigente.

\section{II.- CONTENIDO}

\section{II.1.- Derechos fundamentales y derechos humanos}

Previamente es necesario que debamos exponer la correspondiente distinción entre el concepto de derechos humanos y la que corresponde a los derechos fundamentales, basado en que con frecuencia suelen confundirse ambas denominaciones. Comenzamos señalando que el origen de dichas confusiones tienen algún fundamento macro por la inapropiada denominación de algunas instituciones destinadas a la tutela de determinados derechos como lo es para citar un solo caso: la denominación de Corte Interamericana de Derechos Humanos, siendo que en realidad dicha institución tiene por objeto resolver controversias derivadas de la presunta violación de derechos fundamentales y no precisamente de los derechos humanos propiamente dichos, habida cuenta que estos últimos se relacionan más bien con un nivel de normas de orden moral, carente de un efectos coercitivos que si tienen las normas jurídicas.

Precisamente nuestra actual Constitución Política no utiliza la denominación de Derechos Humanos cuando realiza el catálogo de lo que se denominan más bien Derechos Fundamentales (Título I, Capítulo I), de manera que aparece conveniente realizar la correspondiente disquisición terminológica, habida cuenta la frecuencia con que nos vamos a referir al ámbito de los Derechos Fundamentales en este trabajo.

Ya hemos señalado que la denominación de Derechos Fundamentales utilizado por la Constitución Política del Estado (Capítulo I), en lugar de Derechos Humanos, y ello entendemos que tiene correlación al concepto que expone la moderna doctrina de los Derechos Fundamentales, cuya precisión terminológica la hemos encontrado expuesta de un modo didáctico en el libro Lecciones de Derechos Fundamentales, donde se expone la serie de razones prácticas y teóricas que aconsejan emplear la terminología expuesta para referirse a este concepto (derechos fundamentales) que son:

a) Es más precisa que la expresión de derechos humanos y carece del lastre de la ambigüedad que éste supone; b) Los derechos fundamentales expresan tanto una moralidad básica así como una juricidad básica; c) Las tradiciones lingüísticas de los juristas atribuyen al término "derechos fundamentales" esa dimensión vinculándola a su reconocimiento constitucional 
o legal; y d) Ciñe la estipulación del sentido a la faceta de la pertenencia al Ordenamiento. (Peces, 2004 p. 28). I

Ello implica resumiendo, que todo derecho fundamental corresponde a un derecho humano, pero que no todo derecho humano alcanza la categoría de un derecho fundamental, ello debido básicamente a un tema de positivización que siempre lo ha de tener un derecho fundamental, a diferencia del derecho humano propiamente dicho, de modo restrictivo, que más parece pertenecer al ámbito de la moral y a las normas de esta categoría, que al ámbito jurídico en sí, con la consecuente diferenciación entre ambos conceptos.

El catálogo de los derechos fundamentales, llamados de primera generación o primer grado, que determina el Título I, Capítulo I de la Constitución Política vigente, menciona a la vida, (Artículo 2 inciso 1), a la libertad de tránsito por el territorio nacional (Artículo 2 inciso 11), a trabajar libremente, con sujeción a ley (Artículo 2 inciso 15), siendo que precisamente, la positivización de todos estos derechos, es lo que los distingue de la denominación más amplia de derechos humanos, que se han catalogado con el nomen iuris de derechos fundamentales en la Constitución Política vigente conforme se desprende.

\section{II.2.- Los principios y las normas de derecho fundamental}

Ahora bien, habida cuenta a la ostensible posibilidad del cambio de las normas positivas ya sea mediante su derogación y modificación empleando los mecanismos constitucionales previstos para este efecto, y centrados en la realidad de que el Derecho es una obra humana, cabe pues ciertamente la ominosa posibilidad de la manipulación de estos derechos conforme la conveniencia de quienes representen el poder suficiente para realizar dicho cometido mal utilizando las facultades y el mecanismo que la propia constitución determina. Por estos motivos es precisamente que la doctrina internacional ha expuesto la conveniencia de elevar a la categoría de principios a los derechos fundamentales.

Pues bien, los principios, conforme la conceptualización de Alexy, (2007) en la obra Teoría de los derechos fundamentales, determina que "...son normas que ordenan que algo sea realizado en la mayor medida posible, dentro de las posibilidades jurídicas y reales existentes, por tanto los principios son mandatos de optimización..." (p.67-68) de manera que lo que se refleja en la Constitución vigente, siguiendo los razonamientos que cita este autor (p.55) son en realidad enunciados normativos de derecho fundamental que ordenan la realización de algo en la mayor medida posible, dentro de las posibilidades jurídicas y de hecho existentes. Una de las singularidades de los principios, a diferencia de las reglas de derecho es su generalidad, es decir que los principios tienen un grado de generalidad relativamente alto, frente a la reglas que tienen un nivel de generalidad relativamente bajo (p.65).

Es de apreciar que “...en su significación axiológica objetiva, los derechos fundamentales representan el resultado del acuerdo básico de las diferentes fuerzas sociales, logrado a partir de relaciones de tensión y de los consiguientes esfuerzos de cooperación encaminado al logro de metas comunes..." (Pérez, 1988 p.17), conforme en su obra Los Derechos Fundamentales (1988), prosiguiendo este mismo autor que "...en su dimensión subjetiva, los derechos fundamentales determinan el estatuto jurídico de los ciudadanos, lo mismo en sus relaciones con el Estado que en sus relaciones entre sí..." (p. 20-22)ii, vale decir pues que los llamados enunciados de derechos fundamentales consagrados por la Constitución, representan además la facultad de cualquier ciudadano a solicitar la tutela jurisdiccional efectiva frente a cualquier acto que menoscabe esos derechos objetivos. 
Una definición dogmática de los derechos fundamentales determina que:

Son "derechos fundamentales" todos aquellos derechos subjetivos que corresponden universalmente a "todos" los seres humanos en cuanto datos del status de personas, de ciudadanos o personas con capacidad de obrar; entendiendo por "derecho subjetivo" cualquier expectativa positiva (de prestaciones) o negativa (de no sufrir lesiones) adscrita a un sujeto por una norma jurídica; y por "status" la condición de un sujeto prevista asimismo por una norma jurídica positiva, como presupuesto de su idoneidad para ser titular de situaciones jurídicas y/o autor de los actos que son ejercicios de éstas. Ferrajoli (2009 p.19) iii.

Estos enunciados descriptivos presuponen una concepción material de los derechos fundamentales, conforme lo expone el autor Diez Picaso (2008), quien prosigue señalando que lo verdaderamente peculiar de los derechos es su contenido. (p. 38) iv. Este último autor señala que enfrente a la concepción material de los derechos fundamentales se alza la concepción formal de los mismos, para lo cual lo crucial no es su contenido, sino el rango de la norma que lo reconoce. Bajo esta premisa generalizadora, serían entonces derechos fundamentales todas las disposiciones consagradas en la Constitución.

El Tribunal Constitucional peruano ha adoptado una delimitación conceptual de los derechos fundamentales, remitiéndolos más bien como una expresión del conjunto de valores, conforme se aprecia de la sentencia recaída en el Exp. 1042-2002-AA/TC, en cuyo fundamento 2.2 se determina que "...constituyen componentes estructurales básicos del conjunto de orden jurídico objetivo, puesto que son la expresión jurídica de un sistema de valores que por decisión del constituyente informan todo el conjunto de la organización política y jurídica..."v. Aquí debemos enfatizar que el intérprete de mayor jerarquía de la Constitución prefiere señalar que los derechos fundamentales son la expresión jurídica de un sistema de valores y no de un sistema de principios, pretendiendo quizá incidir en el contenido axiológico del concepto valor, es decir que se incide en el mandato de que es lo bueno, dejando de lado el concepto de lo que debe ser, más asociado al concepto de los principios.

Los principios que subyacen al derecho a la vida, al derecho al libre tránsito y el derecho a la libertad de trabajo, según la Ley, y que se hallan expuestos bajo la forma de enunciados normativos de derechos fundamentales en el artículo 2 incisos 1), 11) y 15), contienen mandatos de optimización, reflejados en normas positivas, contienen órdenes que deberían ser realizados en la mayor cantidad posible, dentro de las posibilidades jurídicas y de hecho existentes.

Los referidos derechos se hallan tutelados por el Estado, no solamente mediante esta declaración de enunciados normativos de derechos fundamentales, sino también por las otras normas complementarias que garantizan el ejercicio de estos derechos, así tenemos por ejemplo el Código Penal, el Código Procesal Penal, un Poder del Estado (Judicial), instituciones autónomas tal como el Ministerio Público, así como la Policía Nacional, a efectos de la tutela del derecho a la vida, en su faz negativa, es decir para sancionar a aquellas personas que atenten contra el derecho a la vida o el derecho a la libertad de tránsito de cualquier ciudadano y en la faz positiva, el estado pretende tutelar el derecho a la vida mediante el sistema público de asistencia de la salud en el país.

Ahora bien y si le asignamos la prelación ordinal correspondiente a una suerte de preeminencia de la larga lista de derechos fundamentales enunciados en el citado artículo 2 de la Constitución, denota en principio que el derecho a la vida stricto sensu, tendría una suerte de preeminencia dentro del catálogo de enunciados normativos de derecho fundamental ya expuestos, luego entonces, y sólo después de considerar el derecho a la vida, tendríamos que considerar el derecho al libre tránsito y la libertad de trabajo, dentro de la ley. Sin embargo, si bien en concreto es factible entender de un modo aislado este mensaje enunciativo, no es menos cierto que en abstracto 
resulta difícil, por no decir complicado considerar el derecho a la vida de ese modo aislado, si es que no la consideramos conjuntamente con la libertad de tránsito o con la libertad al trabajo. El derecho a la vida no se concibe fácil de ejercer si tenemos un serio recorte a la prohibición del derecho al libre tránsito o si se presenta un recorte al derecho a trabajar, como viene sucediendo actualmente. En este escenario, se agrega nítidamente otro derecho fundamental adicional recortado: el derecho a la libertad (artículo 2 inciso 24 de la Constitución).

Luego entonces, se aprecia de inmediato que los decretos supremos que disponen la emergencia y consiguiente cuarentena a nivel nacional han recortado ostensiblemente el derecho a transitar libremente, han recortado la libertad de trabajo dentro de la Ley y desde luego también han recortado también el derecho a la libertad en general.

En este escenario, si tenemos entendido que la razón básica del ejecutivo para haber recortado esos derechos fundamentales se entiende que es porque ha aplicado la preeminencia del derecho a la vida que se pretende proteger, por encima de los otros derechos que le secundan en la larga lista de enunciados normativos de derechos fundamentales. Ello se desprende de un modo inequívoco de la redacción del D.S. 044- 2020-PCM el que refleja el derecho a la protección a la salud, del medio familiar y de la comunidad, citando los artículos 7 y 9 de la Constitución y que sustentan, entre otros, las medidas excepcionales que a continuación se pueden leer del texto normativo.

Ahora bien, si razonamos de un modo abstracto, generalizado, difícilmente podríamos concebir el derecho a la salud de un modo aislado, si lo separamos por ejemplo del derecho a transitar libremente o el derecho a trabajar, menos aún dentro de una economía marcadamente informal, dentro de un país en el que un $65.7 \%$ por ciento de los trabajadores, corresponden a la tasa de empleo informal (Informe del Inei, en Internet)vi.

Pues bien, de este modo tenemos muy claro que en este escenario se ha producido un conflicto de los principios de derecho fundamental que sustentan el derecho a la vida, al transitar libremente y a la libertad para trabajar, incluso también el que corresponde al derecho fundamental a la libertad en general. De esta manera entramos a verificar cómo es que en el presente caso, motivado por una pandemia, se ha producido un evidente conflicto de los principios de derecho que sustentan los diferentes enunciados de derecho fundamental que consagra la Constitución Política vigente.

El autor que venimos siguiendo en algunos segmentos de este comentario, Robert Alexy (2007), prefiere denominar colisión en lugar de conflicto que es la denominación que preferimos emplear para describir la disyuntiva presente (p. 71). El conflicto presente nos sirve para distinguir además la diferencia entre el llamado conflicto de reglas de derecho y el conflicto de principios de derecho. El autor citado explica "....un conflicto entre reglas sólo puede solucionarse mediante la introducción en una de las reglas de una cláusula de excepción que elimine el conflicto o mediante la declaración de lo que por lo menos una de las reglas es inválida..." (p.69), luego se agrega que:

Las colisiones de principios deben ser solucionados de manera totalmente distinta. Cuando dos principios entran en colisión... uno de los dos principios tiene que ceder frente a otro. Sin embargo, esto no significa declarar inválido al principio desplazado ni que en el principio desplazado haya que introducir una cláusula de excepción. (Alexy, 2007 p.70).

Esta distinción metodológica será muy útil al momento de argumentar a favor de un criterio de respuesta al conflicto de los derechos fundamentales, motivado por el recorte de algunos de los mismos con motivo de la pandemia del covid-19. Más adelante nos ocuparemos del método que usualmente se utiliza para superar esta colisión de principios de derecho fundamental. 


\section{II.3.- Conflicto de derechos fundamentales en el país, con motivo de la pandemia del covid-19.}

La pandemia del covid-19, de un modo súbito ha generado, desde nuestro criterio un evidente conflicto de enunciados normativos de derecho fundamental en nuestro país. Veamos: una primera lectura del primer decreto supremo (D.S. 044-2020-PCM) que declara el estado de emergencia y la medida excepcional de cuarentena en el país, no menciona expresamente el derecho a la vida, que es en realidad lo que se pretende preservar mediante la protección del derecho a la salud, del medio familiar y de la comunidad, citando incluso los artículos 7 y 9 de la Constitución, que pertenecen al capítulo II del Título I de la Constitución. De este modo, surge un primer esquema argumentativo que pudiera servir de fuente de cuestionamiento acerca de la constitucionalidad de las medidas excepcionales del estado de emergencia decretado.

Antes debemos detenernos en repasar la pretendida preeminencia de las normas de rango constitucional, relativas a los derechos fundamentales. Ello se origina sin lugar a dudas, en lo que la doctrina ha denominado derechos fundamentales de primera generación, y los otros derechos de similar denominación, pero que se hallan situados a continuación de aquél, por así decir, cuando a los derechos económicos y sociales se les denomina comúnmente derechos fundamentales de segunda generación.

La razón de esta pretendida preeminencia, tiene mucho que ver con la génesis de estos derechos, mientras los primeros, los llamados derechos fundamentales de primera generación, derechos de la persona, tienen un precedente en la Declaración de los Derechos del Hombre y del Ciudadano de la Revolución Francesa de 1789 y en nuestro país hallamos un antecedente similar en el Estatuto Provisorio de San Martín de 1821; en cambio los derechos económicos y sociales se han positivizado recién en el siglo XX, si nos remitimos a la Constitución que produjo la Revolución Mexicana en el año 2017 y posteriormente en la Constitución de Weimar, Alemania, de 1919. En nuestro país, la Constitución de 1920, fue la primera en incorporarlas a su texto normativo.

Alexy (2007), denomina principio constitucional de primer rango cuando tiene capacidad de limitar un derecho fundamental garantizado sin reserva (pág.111). Prosigue explicando el citado autor que tiene rango constitucional de segundo grado sólo si este principio puede limitar un derecho fundamental, conjuntamente con una norma de competencia estatuida en una disposición de reserva. Como podemos verificar este criterio se aleja del criterio genético de los derechos fundamentales para establecer otro criterio de distinción entre derechos fundamentales: los mismos que serían de primer y segundo grado de acuerdo a su propia autonomía conforme se desprende.

La Constitución Política vigente comienza enumerando lo que conforme nuestro criterio constituyen los enunciados normativos de derecho fundamental de primer grado en el Título I, Capítulo I de del texto constitucional, dedicado a exponer los derechos a la persona, habiendo comenzando a definir que la defensa de la persona humana y el respeto de su dignidad es el fin supremo de la sociedad y el Estado (artículo 1). Pues bien, esos derechos de la persona señalándose que:

Suponen el mínimo que se debe reconocer a cada persona como miembro de la sociedad, en su calidad de individuo. En este sentido, para entrar en relación con otros, cada ser humano debe tener garantizado el derecho a la vida, a su identidad, a su honor, intimidad, convicciones, etc. Sin ello es absurdo pensar que un individuo pueda ingresar a una relación social con sus semejantes. Son prerrequisitos indispensables para que pueda existir una interrelación en la sociedad. (Bernales, 2012 p.197).

Sólo después de agotar la citada enumeración de derechos fundamentales de primer grado o de primera generación, la Constitución prosigue enumerando los llamados derechos fundamentales de segunda generación o de segundo grado, Capítulo II del Título I, denominados también derechos 
económicos y sociales más adelante, refiriéndose a los derechos sociales y económicos prosigue señalando que:

También debe regularse el cómo y el cuánto de las relaciones de las personas entre sí, es decir, qué derecho se tiene frente a otro y cuánto pude ser ejercido frente a él. A la inversa, que derecho y cuánto pueden él o los otros ejercer sus derechos frente a sí mismo. vii (Bernales, 2012 p.197).

De este modo, el catálogo de los derechos fundamentales que consagra la norma fundamental vigente, otorga preeminencia, a la vida, a la libertad para transitar y a la libertad para trabajar, situándolos como derechos fundamentales de primera generación o de primer grado y sólo después consagra de ellos, consagra el derecho a la salud y la seguridad social, como parte de los derechos sociales y económicos, llamados también derechos fundamentales de segunda generación o de segundo grado.

Desde este punto de vista, resulta entonces que el Decreto Supremo que ordena el estado de emergencia y la imposición de medidas excepcionales de cuarentena a nivel nacional, se ampara en normas de los llamados derechos fundamentales de segunda generación o segundo grado (artículos 7 y 9 de la norma fundamental) y los coloca por encima de ciertos derechos fundamentales de primera generación o de primer grado, como son el derecho a la libertad para transitar y la libertad para trabajar (artículo 2 incisos 11 y 15), incluida además el derecho a la libertad en general (artículo 2 inciso 24).

Este es un primer argumento simplista que a su vez se desprende de los anteriores razonamientos en relación a la presunta preeminencia de los derechos fundamentales, bajo el argumento, igual de simplista, de que un derecho de segunda generación o segundo grado no podría estar por encima o antes si se quiere, de otro derecho fundamental de primera generación o de primer grado. Ahora bien, ¿podemos razonar de este modo?, desde luego que sí basados en el argumento del orden, teniendo en cuenta además que conforme lo explica Rolla (2002) en su Libro Derechos Fundamentales y Justicia Constitucional que “...no es ajeno el hecho que otras constituciones reconocen la preeminencia de las normas relativas de los derechos fundamentales frente a las otras disposiciones constitucionales..." (pág. 124), quien cita como ejemplo la Constitución Turca, donde se reconoce una posición de supremacía jerárquica a las normas contenidas en el preámbulo. viii

Distinta es la posición de lo que determina la Constitución peruana, de cuya redacción del artículo 3, se desprende la obligatoriedad de realizar una interpretación extensiva de los textos constitucionales, puesto que precisamente dicho texto, semánticamente pareciera sugerirnos lo contrario a la idea de un orden prevalente de las normas de derecho fundamental al determinar que la enunciación de los derechos establecidos en este capítulo no excluye los demás que la Constitución garantiza, ni otros de naturaleza análoga o que se fundan en la dignidad del hombre, o en los principios de soberanía del pueblo, del Estado democrático de derecho o de la forma republicana de gobierno.

De este modo, se determina además la llamada lectura sistemática-llámese también interpretación sistemática- de la Constitución, siendo que la determinación de los derechos personales no esté circunscrita únicamente a los enunciados en el artículo 2 , sino que, además, deben considerarse como tal a todos los otros preceptos constitucionales que la Constitución garantiza. Al respecto, podemos mencionar a manera de ejemplo el artículo 139 inciso 14) de la norma fundamental que determina el derecho a la defensa, el mismo que sin duda tiene el carácter de un derecho fundamental de carácter personal, sujeto además a todas las garantías que tanto la constitución, así como las demás leyes subordinadas, tienen la obligación de cautelar y garantizar. 
Así lo expresa el Tribunal Constitucional mediante lo resuelto en el Exp. 1417-2005- PA-TC, cuando señala que los derechos fundamentales enumerados en el artículo 2 de la Constitución ".... no excluye los demás derechos reconocidos en el texto constitucional ...", posibilitando de este modo la llamada interpretación extensiva del texto constitucional, pudiendo adoptar este carácter casi cualquier otro dispositivo constitucional, incluso también implica el reconocimiento de aquellos otros derechos fundamentales que sin estar explícitamente catalogados dentro de los preceptos constitucionales merecen protección o tutela, se determina que:

Nuestra Constitución Política reconoce, en su artículo $3^{\circ}$ una "enunciación abierta" de derechos fundamentales que, sin estar en el texto de la Constitución, surgen de la dignidad del hombre ..., así el derecho a la verdad, aunque no tiene un reconocimiento expreso en nuestro texto constitucional, es un derecho plenamente protegido derivado en primer lugar de la obligación estatal de proteger los derechos fundamentales y de la tutela jurisdiccional ... o en los principios de soberanía del pueblo, del Estado Democrático de Derecho o de la forma republicada de gobierno. (Exp. 2488- 2002-HC/TC)

Lo cual significa además que el reconocimiento y protección de estos derechos se halla encomendado a los distintos juzgados constitucionales. En similar sentido se ha pronunciado la decisión adoptada en el Exp. 0895-2001-AA/TC (fundamento 5) del Tribunal Constitucional donde luego de establecer que el artículo 3 de la Constitución recoge una "enumeración abierta" de derechos, lo cual no es óbice para sostener que en ciertos derechos constitucionales explícitamente reconocidos, subyacen manifestaciones del derecho que anteriormente no habían sido previstas. Se señala además pertinente que el Tribunal Constitucional deba considerar el desarrollo de los derechos constitucionales expresamente reconocidos, mediante las decisiones que permitan robustecer el respeto a la dignidad del hombre, pues de este modo se evitaría la tendencia a reconocer constantemente a la cláusula constitucional de los derechos "no enumerados" y, con ello, alterar el propósito para el cual fue creada.

Ahora bien, en nuestro caso de pandemia generalizada, se ha producido un conflicto de derechos fundamentales explícitamente establecidos, de manera que no es necesario, por lo menos así lo consideramos, recurrir a ningún derecho subyacente o no enumerado, lo que abre un escenario en el que a la manera de los principios del derecho de donde provienen, deberían encontrar una solución interpretativa ponderada conforme corresponde.

\section{II.4.- Ponderación de derechos fundamentales}

El ámbito de conflicto de enunciados normativos de derechos fundamentales que han generado de un modo ostensible los sucesivos decretos supremos que han recortado diversos derechos fundamentales, bajo la premisa de protección de la salud, deviene ser resuelta mediante el método de la ponderación de los principios de derechos fundamentales en conflicto.

Ya hemos adelantado, citando a RobertAlexy que el conflicto de principios de derecho fundamental deben ser solucionados de manera muy diferente al de los conflictos entre reglas de derecho.

La relación de tensión que supone la concurrencia de los principios en colisión puede solucionarse mediante la llamada ponderación de los intereses contrapuestos, siendo que conforme la exposición de Robert Alexy (2007) esta ponderación supone el establecimiento de cuál de los intereses, que tienen el mismo rango en abstracto, posee el mayor peso en el caso concreto. (p. 72).

El escenario de la ponderación de principios que se ha de realizar para el caso actual, a la luz de los presupuestos doctrinarios expuestos así como los de la jurisprudencia del Tribunal Constitucional deviene una operación compleja, habida cuenta la colisión de principios de distinta gradación por así decirlo y que en apariencia denota que los decretos supremos que ordenan la cuarentena obligatoria 
de los ciudadanos ha adoptado un escenario a favor de derechos de segunda generación (la salud, la familia, la comunidad) en desmedro de otros que corresponden a derechos de primer grado o de primera generación (libertad para transitar, libertad para trabajar).

Ello implica que los argumentos en favor o en contra de las drásticas medidas adoptadas, tendrán que sustentar las razones por las que el presente caso se tenga que determinar cuál de los intereses en conflicto (derechos fundamentales), refleja mayor trascendencia en el caso concreto.

Siempre siguiendo la exposición de Robert Alexy (2009), se determina que entre la teoría de los principios y el principio de proporcionalidad existe conexión, lo que en buena cuenta implica que la llamada proporcionalidad deviene en un principio de la ponderación (p. 95). De este principio se subdividen, como bien lo señala el autor, los sub principios de idoneidad, necesidad y finalmente la que corresponde a la proporcionalidad en sí misma.

El procedimiento de ponderación presupone entonces adoptar un análisis sucesivo lógico comenzando por analizar la idoneidad del recorte de ciertos derechos fundamentales dispuesta; luego deberá procederse a examinar la necesidad de la medida adoptada para finalmente realizar el llamado test de proporcionalidad propiamente dicho entre ambos sub principios, de modo que se pueda llegar a una solución razonada y sobre todo fundamentada.

A efectos de poder explicar, resumidamente el contenido de cada uno de estos sub principios, debemos señalar que el autor Manuel Atienza (2018) co-autor del libro titulado Un debate sobre la ponderación, hace notar que esta técnica tiene una gran alcance en diversos tribunales latinoamericanos -especialmente en cortes supremas y tribunales constitucionales- (p.18), para luego agregar que concretamente en nuestro Tribunal Constitucional peruano, el esquema alexiano (se refiere a Robert Alexy) parece haber tenido un amplio uso. ix

Bajo esta premisa conviene entonces repasar cuál es el esquema del procedimiento de ponderación que el referido autor ha expuesto, y que conforme se ha expuesto, es el que sigue regularmente nuestro Tribunal Constitucional. De esta forma se considera pertinente reproducir el esquema resumido de dicho procedimiento alexiano, que el mismo autor (Atienza) expone en la obra ya citada: comienza remarcando que los dos primeros sub principios se refieren a la optimización en relación con las posibilidades fácticas; significa que una medida (una ley, una sentencia, etc.) que limita un derecho (un bien de considerable importancia) para satisfacer otro, debe ser idónea para esa finalidad y necesaria, o sea, no debe ocurrir que la misma finalidad pudiera alcanzarse con un coste menor. El tercer sub principio (la ponderación o proporcionalidad stricto sensu), por el contrario, tiene que ver con la optimización en relación con las posibilidades normativas. La estructura de ponderación de Alexy -prosigue señalando el autor citado - consta de tres elementos: la ley de la ponderación, la fórmula del peso y las cargas de la argumentación. (p. 18-19).

Ahora bien, todo este esquema teórico que estamos exponiendo resumidamente, tendrá mucho que ver con el escenario procesal para el uso de las garantías procesales y sustantivas que el derecho ha previsto para al acceso a la tutela jurisdiccional de los derechos fundamentales; más aún dentro del escenario de derechos presuntamente conculcados a decenas de miles de personas naturales y jurídicas del país, cuyo conflicto se avecina hipotéticamente en el futuro inmediato.

Ya hemos sido testigos de las primeras críticas que se han realizado a nivel global contra los esquemas de cuarentena que se han decretado u omitido en decenas de países, las primeras de ellas sobretodo en Latinoamérica y el conflicto de pareceres que ya ha producido no pocos agravios verbales y escritos teniendo a la vista las decenas miles de vidas y puestos de trabajo perdidos en el país y el resto del mundo a lo largo del presente estado de emergencia. 


\section{II.5.- La jurisprudencia precedente de los distintos tribunales peruanos}

El tema del conflicto de derechos fundamentales es un asunto que tiene precedentes expresados por la jurisprudencia práctica de nuestro país y ello nos permite repasar estos puntos de vista, que si bien se han pronunciado en razón de conflictos diversos, diferentes a los que son materia de la presente pandemia del covid-19, a la hora del hipotético debate, serán fuente de consulta obligatoria.

El debate acerca del criterio de proporcionalidad que se aplique al caso concreto, entre otros, resulta obligatorio cuando se trata de recortar un derecho fundamental, es decir que tiene carácter vinculante, conforme lo determina la Casación N. 626-2013 Moquegua, (considerando vigésimo cuarto) ampliamente conocido en el país, con motivo del examen de los motivos fundamentados de la medida de prisión preventiva. El considerando vigésimo segundo de la citada resolución casatoria, de un modo resumido expone los sub principios de idoneidad, necesidad y proporcionalidad en sentido estricto, que debe fundamentar el requerimiento fiscal de prisión preventiva, reiterando que el debate sobre este extremo es de carácter obligatorio en toda circunstancia similar.

\section{II.5.1 EL CASO PROFA}

El Tribunal Constitucional ha delineado los criterios que adopta en la aplicación del principio de ponderación, a partir de la resolución adoptada en el Exp. 0025-2005-PI/TC de 25 de abril del 2006, en el llamado caso Profa, originado por la demanda de declaración de inconstitucionalidad del requisito establecido en un reglamento del extinto Consejo Nacional de la Magistratura, por la exigencia a los postulantes a los concursos para plazas de jueces y fiscales a nivel nacional, en acreditar haber aprobado previamente los cursos de formación de la Academia de la Magistratura (Profa).

En el fundamento 2.2. de la referida resolución se señala que el análisis de la norma impugnada bajo el principio de proporcionalidad (ponderación) debe efectuarse realizando los siguientes pasos:

a) Determinación del tratamiento legislativo diferente: la intervención en la prohibición de discriminación.

b) Determinación de la "intensidad" de la intervención en igualdad.

c) Determinación de la finalidad del tratamiento diferente (objetivo y fin).

d) Examen de idoneidad.

e) Examen de necesidad.

f) Examen de proporcionalidad en sentido estricto o ponderación.

Es decir que el Tribunal Constitucional, antes de fundamentar los sub principios señalados por la doctrina de los derechos fundamentales (idoneidad, necesidad y proporcionalidad propiamente dicha), se aparta del esquema alexiano, hipotéticamente con el propósito de sustentar el fallo final, y para ello se determinó que era necesario fijar los grupos en los que se subdivide la intervención presuntamente discriminatoria: para ello seguimos casi textualmente lo que enuncia la resolución en comentario:

a) -abogados sin Profa- $y$-abogados que aprobaron el curso del Profa, paso a).

b) La intensidad de la intervención en igualdad de condiciones del acceso a la función pública en tres niveles: -intensidad grave-, -intensidad media-, -intensidad leve-, habiendo calificado que se trataba de una intervención grave, porque incidía en un derecho constitucional que es el acceso a la función pública,

c) El criterio de la finalidad del tratamiento es una variante compleja que comprende dos objetivos: el objetivo y el fin, siendo el objetivo el estado de cosas o situación jurídica que el legislador pretende conformar a través del tratamiento diferenciado mientras la finalidad o el 
fin viene a ser el derecho, principio o bien jurídico cuya realización u optimización se logra con la conformación del objetivo.

Pues bien, conforme nuestro criterio, con lo expuesto en el paso b), el Tribunal Constitucional prácticamente ya había resuelto la controversia sin necesidad siquiera de sustentar los sub principios siguientes (idoneidad, necesidad y proporcionalidad stricto sensu), porque se consideraba la intervención diferenciada del emisor de la norma cuestionada (en este caso, el extinto CNM) como una intervención a la igualdad de condiciones para el acceso a la magistratura que calificó de grave por incidir en un derecho constitucional, definiendo así por anticipado la controversia. Si ello ya era así, -grave intervención a la igualdad de condiciones- poco o nada de lo sustentado en adelante podría ya revertir esta consideración lapidaria, o sea que, siempre bajo nuestro criterio, el resto de los argumentos analizados por el Tribunal Constitucional, devinieron en pura retórica, destinada a sustentar la decisión expresamente adelantada en este considerado.

En el examen de idoneidad (fundamento 2.2.4) que realiza el Tribunal Constitucional, desvía el razonamiento a un tema divergente de la controversia misma y termina aceptando que el requisito del PROFA, constituye un medio adecuado para la conformación de una magistratura idónea, para luego nomás y de un modo incongruente considerar que éste es un principio relativo condicionado a las características y nivel del PROFA, (obsérvese la evidente contradicción del razonamiento) pues en lugar de ocuparse del mandato de optimización con relación a las posibilidades fácticas que contenía o no el acto normativo cuestionado de inconstitucionalidad, y como acota Manuel Atienza (p.18), el tema de la idoneidad determina que la medida que limita el derecho para satisfacer otro, deba ser idónea para obtener esa finalidad. El Tribunal relativiza el tema de la idoneidad de la medida adoptada de requerir el curso del Profa a los postulantes a pesar de que en el mismo texto resolutivo se admite que el requisito del Profa constituye un medio adecuado para la conformación de una magistratura idónea.

En el examen de necesidad (fundamento 2.2.5) el Tribunal Constitucional determina que el examen debe comprender dos aspectos: (1) la detección si hay medios hipotéticos alternativos idóneos y (2) la determinación de, (2.1) si tales medios -idóneos- no intervienen en la prohibición de discriminación, o (2.2) si, interviniéndolo, tal intervención reviste menor intensidad. Prosigue el Tribunal explicando su razonamiento mediante la cita de un precedente: "Conforme se precisó, dos aspectos han de analizarse bajo este principio: a) Si existen medios alternativos igualmente idóneos para la realización del objetivo y, b), si tales medios no afectan el principio de igualdad o, de hacerlo, la afectación reviste menor intensidad que la del cuestionado. A continuación se determina con amplia extensión la existencia de los otros medios presuntamente idóneos para la garantizar la formación de los magistrados ya nombrados, sustentando la no necesidad de afectar el derecho de igualdad entre los postulantes a los cargos de jueces y fiscales a nivel nacional.

El Tribunal Constitucional resuelve que el legislador disponía de al menos de un medio alternativo para alcanzar el objetivo propuesto (la idoneidad de los magistrados en función) sin afectar el derecho de acceso a la función pública, sin embargo, contrariamente, razonó que un medio requerido -el requisito del PROFA- que si bien alcanza el objetivo de idoneidad de la magistratura pero que afecta el derecho a la no discriminación. En consecuencia -concluye el Tribunal- en tanto la exigencia del requisito del PROFA para postular a la magistratura no supera el test de necesidad, ella es inconstitucional, por consiguiente no es menester examinar el sub principio de ponderación o proporcionalidad en sentido estricto.

Las incongruencias saltan a la vista, por un lado, el Tribunal Constitucional admite que la Academia de la Magistratura contribuye a lograr la idoneidad en los magistrados del país, para sin embargo, en la misma resolución relativiza el sub principio de idoneidad bajo el argumento de que no se ha demostrado que el PROFA tenga los niveles de idoneidad para lograr este objetivo. Este 
argumento determina al Tribunal que afirme que no es necesario afectar el derecho a la igualdad de oportunidades de acceso a la función pública porque existen medios alternativos idóneos para la realización del objetivo. La pregunta salta a la vista ¿No es que el Tribunal Constitucional, en la misma sentencia, argumentó sin mayor sustento que el PROFA no garantizaba la formación adecuada de los magistrados?.

Conforme podemos verificar, no es factible obtener líneas interpretativas congruentes en la sentencia analizada, por lo menos no en el tema del examen del principio de ponderación. Esta sentencia del Tribunal Constitucional, deja abierta la posibilidad de un análisis más detallado acerca de sus implicancias más notorias, la primera de ellas, el implícito rechazo a la idea de que magistratura sea una especialidad del Derecho, la misma que tenga que lograrse previamente. De este modo se otorga implícitamente una presunción de eficacia a las Universidades del país, en la formación de futuros jueces y magistrados (que no es su función precisamente) y de un modo paradójico, le regatea esta eficacia a la Academia de la Magistratura, al grado de relativizar la garantía para llevar adelante el objetivo de lograr la idoneidad de los magistrados del país. La idea central de esta sentencia y acaso la más controvertida, es aquella que desliza el propósito de que los magistrados del país, tengan que aprobar los cursos necesarios para el desempeño de tan delicada función, sólo después de haber sido nombrados para el cargo. De este modo y conforme nuestro criterio personal, este precedente del Tribunal Constitucional no será de ninguna utilidad en el hipotético escenario de colisión de derechos fundamentales que se viene produciendo con motivo de la cuarentena obligatoria por motivo de la pandemia por el covid-19.

\section{II.5.2 EL CASO ASOCIACIÓN UNIDOS CENTENARIO Y OTROS DE HUARAL}

En la resolución emitida en el Exp. 8726-2005 PA/TC de 24 de noviembre del 2005, con motivo del recurso de agravio constitucional interpuesto por la Asociación Unidos Centenario y otros de Huaral, que había solicitado la inaplicabilidad de una Ordenanza Municipal del Alcalde Provincial de Huaral, alegando entre otros, una lesión a la libertad del trabajo, a la salud, motivó un pronunciamiento del Tribunal que involucra la ponderación de principios de derecho fundamental antes referidos.

Este precedente resulta siendo interesante si consideramos el tema debatido en torno a los derechos fundamentales involucrados en el caso: derecho a la libertad de trabajar, derecho a la salud, que en tiempos más recientes han entrado igualmente en colisión con motivo de la pandemia del covid-19.

El problema se originó por la emisión de la Ordenanza Municipal № 006-2004, que no permitía el trabajo las 24 horas del día en un área que ocupaban los demandantes en la ciudad de Huaral, prohibiendo en las inmediaciones el comercio ambulatorio, bajo pena de decomiso. Este mandato, lesionaba, conforme argumentaron los demandantes, el derecho a la libertad para trabajar, entre otros, de manera que la sentencia emitida involucra argumentos muy interesantes acerca de este derecho fundamental.

La sentencia comienza sustentando (fundamento 6) que el comercio ambulatorio está comprendido bajo el ámbito de protección a la libertad para trabajar, el mismo que constituye un derecho fundamental conforme lo señala el artículo 2 inciso 15 de la norma fundamental y que el contenido o ámbito de protección de este derecho fundamental constituye el libre ejercicio de toda actividad económica. Luego, citando un precedente del Tribunal Constitucional Alemán, concluyen el argumento señalando que el contenido de la libertad para trabajar puede ser entendido como la facultad de ejercer toda actividad que tenga como finalidad el sustento vital de una persona.

Prosigue el fundamento 7 de la sentencia bajo comentario, la misma que señala que el trabajo en cuanto derecho fundamental detenta una doble faz. Por un lado, constituye derecho de defensa y, 
por otro, derecho de protección. En cuanto derecho de defensa, proyecta su vinculatoriedad típica, clásica, oponible al Estado y a particulares, como esfera de actuación libre. En cuanto derecho de protección, la liberta de trabajo reconoce a la persona el derecho a una acción positiva, que vincula al Estado a la protección activa del bien jusfundamental protegido -libre trabajo- a través del establecimiento de normas, procedimientos e instituciones orientadas a hacer posible el derecho de tal derecho fundamental.

Hemos resaltado estos argumentos en razón de su particular incidencia del recorte a este derecho fundamental -libertad de trabajo- que se viene produciendo en la actualidad con motivo de la cuarentena obligatoria decretada por el Poder Ejecutivo, que en buena cuenta implica la limitación absoluta, entre otros, del comercio ambulatorio, resaltando la solidez del argumento en cuanto vincula al Estado con la protección y garantía para el ejercicio de este derecho fundamental.

En los fundamentos 11 y 12 de la referida sentencia, se realiza una descripción del objetivo y finalidad de la intervención en la libertad para trabajar, en esa ocasión con la claridad y pertinencia suficientes a efectos de poderse considerar un precedente dotado de la debida congruencia y pertinencia para ser citado como argumento en favor de determinada postura del conflicto.

El Tribunal determinó que la finalidad de la intervención comprende la finalidad que el órgano productor de la norma ha pretendido alcanzar a través de la medida implementada. Así como que para el presente caso, tal medida suele ser denominada como "intervención" en la estructura del principio de proporcionalidad. Ahora bien, prosigue, la finalidad implica, a su vez, dos aspectos: el objetivo y el fin. El objetivo es el estado de cosas que pretende lograrse con la medida (intervención) normativa. El fin es el derecho, principio o valor constitucional que justifica dicha intervención.

La idoneidad de la ordenanza impugnada por los demandantes es analizada en los considerandos 18 y 19 de la sentencia bajo comentario, siendo que el Tribunal determina que se trata de inquirir acerca de si la medida adoptada, esto es, la prohibición del comercio ambulatorio, es adecuada o conducente al objetivo de la Ordenanza impugnada concluyendo que se puede responder afirmativamente en tanto que el Municipio emplazado buscaba proteger el orden y el ornato del Centro Histórico de la ciudad de Huaral. Más adelante la misma sentencia determina que la declaración de zona rígida de los centros históricos de las ciudades es una medida que ha sido considerada como un medio conducente o idóneo para la consecución de un centro histórico con orden, ornato y protegido. Es de advertir que esta declaración bien puede ser aplicada a todas las ciudades del país, por lo menos a lo que se denomina centro histórico.

Respecto al examen de necesidad de la ordenanza cuestionada, los considerandos 20 y 21 de la sentencia en comentario se ocupan de argumentar de un modo congruente, la procedencia de este requisito de la ponderación. Citando un precedente, indicaron que el Tribunal Constitucional señaló que mediante este examen se analiza si existen medios alternativos al optado por el legislador que no sean gravosos o, al menos, que lo sean en menor intensidad. Se trata de un análisis de una relación medio-medio, esto es de una comparación entre medios, el optado por el legislador y el o los hipotéticos que hubiera podido adoptar para alcanzar el mismo fin. Por esto, el o los medios hipotéticos alternativos han de ser igualmente idóneos. La respuesta fue negativa en razón de que cualquier medio alternativo hipotético que pudiera plantearse tendría que admitir previamente la posibilidad de actividades comerciales en la vía pública, pero tal no es el caso.... En síntesis, la consecución del objetivo trazado sólo puede alcanzarse a través de la restricción de la libertad para trabajar en las vías públicas, si es que tal interdicción tiene lugar en el denominado el "centro histórico" de la ciudad, tal como se desprende del texto de la resolución bajo comentario.

Finalmente, el examen de ponderación realizada por el Tribunal concluye señalando que la medida, conforme al test de ponderación o proporcionalidad en sentido estricto, se establece 
una relación según la cual cuanto mayor es la intensidad de la intervención de la libertad de trabajo, tanto mayor ha de ser el grado de realización u optimización del fin constitucional. Si tal relación se cumple, entonces la intervención en la libertad de trabajo habrá superado el examen de ponderación y no será inconstitucional; por el contrario, en el supuesto de que la intensidad de la afectación en la libertad de trabajo sea mayor al grado de realización del fin constitucional, entonces, la intervención en dicha libertad no estará justificada y será inconstitucional, tal como se señala. En el considerando 23 de la resolución se determina que la intensidad de la intervención en la libertad para trabajar es leve mientras que el grado de optimización o realización del fin constitucional (orden público, protección del patrimonio cultural de la nación) es elevado. Es decir, en la intervención examinada, mientras el grado de optimización del orden público y el patrimonio cultural es elevado, la intensidad de la intervención en la libertad para trabajar es leve. En el considerando 25 el Tribunal examina el mismo asunto, pero en forma negativa, es decir que la intensidad en la intervención en la libertad para trabajar es leve porque los miembros de la asociación recurrente pueden ejercer su libertad de trabajo en forma regular en los lugares y centros que al efecto establece la Municipalidad. Por el contrario, el Tribunal determina que la no realización de los fines constitucionales es de elevada intensidad debido a que: a) el desorden puede afectar un tránsito ordenado, seguro, sin peligro para los transeúntes y los propios vendedores ambulantes, y b) el deterioro del ornato puede comprometer el patrimonio cultural histórico de la ciudad de Huaral, conformada justamente por el centro histórico de dicha ciudad.

Así como se puede verificar, el procedimiento simple de seguir un lineamiento doctrinario de razonamiento para argumentar, sin añadidos inadecuados, determinan una sentencia congruente y razonable conforme se puede verificar.

\section{III.- Argumentos para la ponderación de derechos fundamentales con motivo de las normas que las han recortado con motivo de la pandemia del covid-19.}

Este escenario de conflicto de derechos fundamentales suscitado entre los comerciantes ambulantes de la ciudad de Huaral y el Municipio de la citada localidad, que ha sido descrito precedentemente, deviene similar en algún extremo con el hipotético escenario de afectación de derechos fundamentales con motivo de la pandemia del covid-19, e invita proponer una enumeración tentativa de los argumentos que habrán de debatirse en el caso hipotético, conforme se puede verificar:

a) El problema de fondo reside en la afectación del derecho a la libertad para transitar y la libertad para trabajar (artículo 2 incisos 11 y 15 de la Constitución, en base a la presunta preeminencia del derecho a la salud, el medio familiar y la comunidad (artículos 7 y 9 de la Constitución). De este modo, a efectos del escenario que vamos a describir a continuación, nos situamos en el hipotético caso de alguna controversia en la vía constitucional, la misma que se habría de sustentar en la presunta afectación de determinados derechos fundamentales.

b) El primer escenario posible en este hipotético conflicto de derechos fundamentales, es que el ejecutivo ha otorgado preeminencia a algunos derechos económicos y sociales, llamados también de segunda generación o de segundo grado por encima de ciertos derechos fundamentales de primera generación o de primer grado, lo cual implica que el fundamento jurídico utilizado por el ejecutivo el recorte de derechos fundamentales, ha sido débil, según nuestro punto de vista, lo que obligará a sus representantes un especial esfuerzo argumentativo al momento de defender la citada preeminencia.

c) En cambio, un argumento fuerte a favor del Ejecutivo sería el haber invocado su función de garantizar el derecho a vivir de (artículo 2 inciso 1 de la Constitución), en lugar del derecho a la salud y a su protección (artículos 7 y 9 de la Constitución), que por lo menos en el plano 
ordinal, se hallan por detrás de los derechos fundamentales al derecho al libre tránsito y a la libertad para trabajar (artículo 2 incisos 11 y 15 de la Constitución).

d) El argumento de la idoneidad de las normas que recortan derechos fundamentales con motivo de la pandemia, habrá de incidir en demostrar si la medida adoptada, recorte de la libertad de tránsito y la libertad de trabajo, es conducente al objetivo de los decretos supremos impugnados, es decir que si estas medidas pueden considerarse un medio conducente idóneo al objetivo invocado cual es el de preservar la salud, el medio familiar y la comunidad.

e) El argumento de la necesidad de las normas que recortan derechos fundamentales con motivo de la pandemia, habrá de incidir en el hecho de verificar si existen otros medios distintos al optado por el legislador que no sean gravosos o, al menos, que lo sean de menor intensidad que del adoptado. Ello implica realizar una diferenciación entre los medios empleados, el optado por el legislador y el o los hipotéticos medios que se hubiera podido adoptar para alcanzar el mismo fin. Este será un punto neurálgico de la discusión conforme nuestro criterio; casi podríamos anticipar que decisivo en la controversia, habida cuenta que ambas partes habrán de sustentar sus argumentos sus posiciones, como por ejemplo, aquella del tradicional y comprobado abandono del sistema de salud y falta de prevención por parte del Estado, que podrá ser fácilmente demostrado ante los Tribunales, o aquel otro del tradicional irrespeto por parte de los pobladores a la autoridad y a las normas de seguridad y protección emitidas por el Estado, que también podrá ser fácilmente demostrado en los Tribunales. Los hipotéticos debates se muestran anticipadamente muy interesantes.

f) En el hipotético caso del conflicto de derechos fundamentales con motivo de la pandemia del covid-19, el examen de ponderación o proporcionalidad propiamente dicha, va a establecer, como bien lo determinó el Tribunal Constitucional en la sentencia del caso comerciantes ambulantes de Huaral, es decir se trata de establecer la relación según la cual cuanto mayor es la intensidad de la intervención de la libertad de trabajo tanto como mayor ha de ser el grado de realización u optimización del fin constitucional. Ello equivale en buena cuenta a señalar si esa intensidad de intervención ha sido leve, moderada o elevada mientras el grado de optimización o realización del fin constitucional (preservar la salud como parte de la política de salud del Estado) es leve, moderado o elevado.

g) Reiteramos que el argumento fuerte del ejecutivo, aunque sólo implícito, en los decretos supremos que ordenan las medidas de cuarentena, lo es el de la protección a la vida humana de los pobladores del país (artículo 2 inciso 1) de la norma fundamental). En este escenario, la preeminencia de los enunciados de derechos constitucionales otorgaría una defensa menos complicada al recorte de los derechos efectuados, aunque el tema de fondo es si será factible una argumentación exitosa en este sentido, habida cuenta que no se invocó expresamente el derecho fundamental a la vida para sustentar los decretos supremos que recortan derechos.

h) El cuestionamiento de los decretos supremos que recortan derechos fundamentales, tendrán mejor carga argumentativa conforme se dirijan contra aquellos que han ido prorrogando las drásticas medidas adoptadas, especialmente contra éste último el D.S. 094-2020PCM, habida cuenta que para la fecha de su emisión, ya se tenían conocimiento de ciertos procedimientos de tratamiento y prevención del covid-19, las mismas que se reflejan en los protocolos de atención para esta enfermedad por parte del Minsa, publicados y oficializados anteriormente a las normas de prórroga de las medidas del estado de emergencia. 


\section{IV.- Conclusiones}

A manera de resumen, podemos citar las siguientes conclusiones del presente trabajo.

IV.1.- Es muy probable, un escenario de controversia por la vía constitucional, por el recorte de los derechos fundamentales de primera generación o de primer grado (derecho a la libertad para transitar y derecho a la libertad para transitar, artículo 2 incisos 11 y 15 de la Constitución), ordenados por los sucesivos decretos supremos emitidos por el Poder Ejecutivo de nuestro país.

IV.2.- El escenario de controversia por la vía constitucional por este motivo, se vislumbra más probable aún y con mejores posibilidades argumentativas contra los últimos decretos supremos de prórroga del estado de emergencia, sobre todo contra este último, el D.S. 094-2020-PCM, habida cuenta que para la fecha de su emisión, ya se tenían conocimiento de ciertos procedimientos de tratamiento y prevención del covid-19, las mismas que se reflejan en los protocolos de atención para esta enfermedad por parte del Minsa, publicados y oficializados anteriormente.

IV.3.- En el hipotético caso de una controversia por la vía constitucional por este motivo, se hará indispensable realizar un examen de ponderación para determinar la preeminencia positiva o negativa de los derechos fundamentales afectados, existiendo un precedente en el Exp. 8726-2005- PA/TC que ha determinado de un modo muy didáctico los alcances argumentativos en los que deberá centrarse el hipotético debate del conflicto de derechos.

IV.4.- El punto neurálgico, medular, que podría decidir el resultado del debate, resulta siendo el examen de la necesidad del recorte de los derechos fundamentales de primer grado decretado por el gobierno, es decir que será necesario verificar si existen medios distintos al optado por el legislador que no sean perjudiciales o, al menos, que lo sean en menor medida. Ello implica que habrá que realizar una diferenciación entre los medios empleados, el asumido por el legislador y el o los hipotéticos medios que hubiera podido asumir el gobierno para alcanzar la misma finalidad.

\section{V.- REFERENCIAS BIBLIOGRÁFICAS}

- Alexy, R. (2007) Teoría de los Derechos Fundamentales, Centro de Estudios Políticos y Constitucionales, 2da. Ed. en castellano, Madrid, España.

- Atienza, M (2018) Un debate sobre la ponderación; Ed. Tribunal Constitucional de Bolivia, Sucre, Bolivia.

- Bernales, B.(2012) La Constitución de 1993, Veinte años después; Lima, Perú: Editorial Idemsa

- Diez Picaso, L.(2008) Sistema de los Derechos Fundamentales, Editorial Aranzadi, 3era. Ed. Pamplona, España,

- Ferrajoli, L. (2008) Los Fundamentos de los Derechos Fundamentales, 4ta. Ed., Madrid, España: Editorial. Trotta

- Rolla, G. (2002) Derechos Fundamentales y Justicia Constitucional, Ed.

- Universidad Autónoma de México, México.

- Tribunal Constitucional del Perú (2020) Consulta de causas por Internet; recuperado de: https://www.tc.gob.pe/consultas-de-causas/. 


\section{Citas Bibliográficas}

I. Alexy, R. (2007) Teoría de los Derechos Fundamentales; Centro de Estudios Políticos y Constitucionales, 2da. Ed. en Castellano, Madrid, España.

II. Pérez, A. (1988) Los Derechos Fundamentales; 3era. Ed. Madrid, España: Tecnos, S.A.

III. Ferrajoli, L. (2009) Los Fundamentos de los Derechos Fundamentales; 4ta. Ed. Madrid, España: Trotta S.A.

IV. Diez Picaso, L. (2008) Sistema de los Derechos Fundamentales; 3era. Ed., Pamplona, España: Aranzadi.

V. Tribunal Constitucional del Perú (2020) recuperado de: https://tc.gob.pe/ jurisprudencia/2003/01042-2002-AA.pdf 23/05/2020.

VI. https://www.youtube.com/watch?v=Ug-pjDI- DOc\&list=RDqvG1XC0qfeQ\&index=12 el 23/05/2020.

VII. Bernales, E. (2012) La Constitución de 1993, Veinte años después, Lima, Perú: Idemsa.

VIII. Rolla G. (2002) Derechos Fundamentales y Justicia Constitucional; Universidad Autónoma de México, México, 1era. Ed. 2002

IX. Atienza, M (2018), Un debate sobre la ponderación; Ed. Tribunal Constitucional Plurinacional de Bolivia; Sucre, Bolivia.

Fecha de recepción: 22 de mayo de 2020

Fecha de aceptación: 01 de junio de 2020 